\title{
Choline Chloride-Lactic Acid-Based NADES As an Extraction Medium in a Response Surface Methodology-Optimized Method for the Extraction of Phenolic Compounds from Hazelnut Skin
}

\author{
Chiara Fanali ${ }^{1, *}$, Valeria Gallo ${ }^{1}$, Susanna Della Posta ${ }^{1}$, Laura Dugo ${ }^{1} \mathbb{D}$, Leone Mazzeo ${ }^{2,3} \mathbb{D}$, Marco Cocchi $^{2}$ (D), \\ Vincenzo Piemonte ${ }^{2}$ (D) and Laura De Gara ${ }^{1}$ (D)
}

check for

updates

Citation: Fanali, C.; Gallo, V.; Della

Posta, S.; Dugo, L.; Mazzeo, L.;

Cocchi, M.; Piemonte, V.; De Gara, L. Choline Chloride-Lactic Acid-Based

NADES As an Extraction Medium in a

Response Surface Methodology-

Optimized Method for the Extraction of Phenolic Compounds from Hazelnut Skin. Molecules 2021, 26, 2652. https://doi.org/10.3390/ molecules26092652

Academic Editor: Joaquín García Álvarez

Received: 13 April 2021

Accepted: 29 April 2021

Published: 1 May 2021

Publisher's Note: MDPI stays neutral with regard to jurisdictional claims in published maps and institutional affiliations.

Copyright: (c) 2021 by the authors. Licensee MDPI, Basel, Switzerland. This article is an open access article distributed under the terms and conditions of the Creative Commons Attribution (CC BY) license (https:/ / creativecommons.org/licenses/by/ $4.0 /)$.
1 Department of Science and Technology for Humans and the Environment, Campus Bio-Medico University of Rome, Via Álvaro del Portillo 21, 00128 Rome, Italy; v.gallo@unicampus.it (V.G.);

s.dellaposta@unicampus.it (S.D.P.); 1.dugo@unicampus.it (L.D.); 1.degara@unicampus.it (L.D.G.)

2 Department of Engineering, Campus Bio-Medico University of Rome, Via Álvaro del Portillo 21, 00128 Rome, Italy; leone.mazzeo@uniroma1.it (L.M.); m.cocchi@unicampus.it (M.C.); v.piemonte@unicampus.it (V.P.)

3 Department of Chemical Engineering Materials \& Environment, Sapienza University of Rome, Via Eudossiana 18, 00184 Rome, Italy

* Correspondence: c.fanali@unicampus.it; Tel.: +39-06-225419471

\begin{abstract}
Deep eutectic solvents (DESs) are promising green solvents for the extraction of compounds from food byproducts. Hazelnut (Corylus avellana L.) is one of the most commonly cultivated tree nuts worldwide. The skin represents one of the major byproducts of the hazelnut industry and accounts for $2.5 \%$ of the total hazelnut kernel weight. It is a rich source of phenolic compounds like flavan-3-ols, flavonols, dihydrochalcones, and phenolic acids. In this work, fifteen DESs based on choline chloride and betaine, with different compositions, were studied in order to test their phenolic compounds extraction efficiency through the determination of their total concentration via Folin-Ciocalteu assay. A qualitative analysis of extracted phenolic compounds was assessed by HPLC with UV and MS detection. Using the DES with the best extraction efficiency, a new ultrasound-assisted solid liquid extraction (UA-SLE) method was optimized though the response surface methodology (RSM), taking into account some extraction parameters. Efficient recovery of extracted phenolic compounds was achieved using a 35\% water solution of choline chloride and lactic acid (molar ratio 1:2) as an extraction solvent, working at $80^{\circ} \mathrm{C}$ and with a solid-to-solvent ratio of 1:25 $\mathrm{gmL}^{-1}$. The optimized conditions made it possible to recover $39 \%$ more phenolic compounds compared to a classic organic solvent.
\end{abstract}

Keywords: hazelnut skin; deep eutectic solvents; phenolic compounds; response surface methodology; ultrasound-assisted solid-liquid extraction

\section{Introduction}

A wide range of dry fruits from deciduous trees, such as almonds, nuts, cashew nuts, hazelnuts, macadamias, pine nuts, pistachios, and walnuts, have been consumed since ancient times as an important source of energy [1]. The Food and Drug Administration (FDA) and European Food Safety Authority (EFSA) recommend a daily consumption of nuts, including hazelnut, for coronary heart disease risk reduction ( $42.5 \mathrm{~g}$ according to the FDA and $30 \mathrm{~g}$ according to the EFSA) [2]. Hazelnut (Corylus avellana L., Betulaceae family) is one of the most commonly cultivated tree nuts worldwide [3]. World hazelnut production was around 1.1 million tons/year in 2019 [4]. Turkey is the leading producer, accounting for about 70\% of total supply, and Italy is the second largest hazelnut-producing area [5]. Hazelnut is characterized by the presence of phenolic compounds such as flavan-3-ols, 
benzoic acids, and flavonols [5]. During processing of hazelnut, a large number of waste products, such as hazelnut skin and other byproducts, including hard shell, green leafy cover, and tree leaf, are generated [6]. The skin represents about $2.5 \%$ of the total hazelnut kernel weight and is usually removed by blanching or roasting in order to improve the kernel flavor, color, and crunch and for the use of the kernel in the confectionery industry and bakeries. Hazelnut skins are used without treatment for animal feed, but several studies have shown the possibility of using hazelnut skins as an inexpensive source of bioactive compounds to apply in nutraceutical, pharmaceutical, or cosmetic products [3]. Hazelnut skins are a rich source of phenolic compounds. According to studies from the literature, the main phenolic subclass is by far the flavan-3-ols, in both their monomeric and polymeric forms, accounting for more than $95 \%$ of the total hazelnut skin phenolic compounds. Flavonols and dihydrochalcones represent an additional $3.5 \%$ while phenolic acids account for less than $1 \%$ of the total identified phenolics. Proanthocyanidins (PAs) are oligomers or polymers, also known as condensed tannins, classified as procyanidins, propelargonidins, or prodelphinidins on the basis of the flavan-3-ol units (epi)catechin, (epi)afzelechin, or (epi)gallocatechin, respectively [1,7]. Phenolic compounds from hazelnut skin are typically extracted using a solid-liquid extraction technique with organic solvents such as methanol, ethanol, and methanol/water mixtures [1,3]. With the emergence of green chemistry, interest in the search for green solvents has grown. Ethanol or water/ethanol mixtures have been used as green conventional solvent for the extraction of phenolic compounds in some food and food byproducts; for example, in chickpeas [8] and grape peels, seeds, and stems [9]. Alternatively, deep eutectic solvents (DESs), which were first identified by Abbott and colleagues [10], have aroused considerable attention due to their remarkable physicochemical properties, such as non-volatility, thermal stability, conductivity, and excellent solubility [11]. DESs are inexpensive, more synthetically accessible, and biodegradable and can be employed in fields relating to electrochemistry, organic reactions, catalysis, nanomaterials, extraction, and separation thanks to their green and excellent physicochemical properties [11]. DESs are mainly mixtures consisting of a hydrogen bond donor (HBD) and a hydrogen bond acceptor (HBA). By simply mixing the HBA component (most often a quaternary ammonium salt or metal salt) with the HBD component (various alcohols, amides, amines, or acids) in a given molar ratio, a homogeneous solvent is usually formed with a melting point lower than the melting points of the pure constituents. When each component that composes the DES is natural, we can define it as a natural deep eutectic solvent (NADES) [12]. Some points regarding the main chemical and physical characteristics of DES can be noted in relation to the nature of HBAs and HBDs. In general, hydrophilic DESs are more viscous when compared to ionic and non-ionic hydrophobic DESs. Furthermore, it has been noted that the viscosity of hydrophilic DESs is influenced more by HBDs. Regarding density, similar points can be made: hydrophobic DESs have densities close to or lower than the density of water while hydrophilic DESs show higher densities [13]. In general, DESs and NADESs are less toxic than most organic solvents, and NADESs are less toxic than DESs. Hayyan et al. conducted a study showing the relationship between the toxicity of NADESs, based on choline chloride, and HBDs [14]. In addition, predictive multitasking quantitative structure-toxicity relationship (mtk-QSTR) in silico studies have provided a ranking of some HBDs in terms of their contribution to the toxicity of the final DES. Among the various HBDs, alcoholic sugars and linear chain alcohols showed a negative contribution to the final solvent toxicity, while sugars and amides provided a medium level of toxicity and, with the exception of lactic acid, the organic and inorganic acids tested had the highest contributions to the final toxicity [15].

Some studies have been published on the extraction of phenolic compounds from both waste and food matrices using DESs, which showed good extraction capacities compared to traditional solvents [16]. Meng et al. evaluated different ChCl-based DESs for the extraction of quercetin, kaempferol, naringenin, and isoramnetin from Pollen Typhae samples [17]. Lakka et al. described the extraction of anthocyanins and flavonols from saffron using 
a DES consisting of lactic acid and glycine in a 5:1 molar ratio [18]. UA extraction of phenolic compounds from olive oil using several DESs and NADESs with $\mathrm{ChCl}$ and betaine has been reported [19]. In 2016, Khezeli et al. reported the extraction of phenolic compounds from almond, demonstrating that DESs prepared with choline chloride $(\mathrm{ChCl})$ combined with ethylene glycol and/or glycerol were better than ethylene glycol or glycerol alone, probably due to the higher degree of hydrogen bonding and the greater number of electrostatic interactions in DESs with target analytes compared to pure ethylene glycol and glycerol [20]. Among the methods developed, some involved food waste matrices. Ozturk et al. conducted a study on orange peel waste samples, testing various DESs composed of $\mathrm{ChCl}$ as the $\mathrm{HBA}$ and ethylene glycol and glycerol as the HBD, in various molar ratios [21]. DESs composed by $\mathrm{ChCl}$ and betaine as the $\mathrm{HBA}$ were also used for the extraction of bioactive compounds from spent coffee grounds, obtaining good results in terms of efficiency [22]. Bonacci et al. tested some NADESs using a microwave-assisted extraction (MAE) approach to obtain phenolic compounds from olive oil processing waste, proving that this is an effective alternative extraction media [23].

To the best of our knowledge, there are no studies in the literature that have tested DESs for the extraction of bioactive molecules from hazelnut skin. The aim of this study was the development of an ecofriendly method for the extraction of bioactive phenolic compounds from hazelnut skin based on solid-liquid extraction using DESs. Obtained extracts from hazelnut skins, using betaine and ChCl-based DESs, were characterized in terms of phenolic compounds content using high performance liquid chromatography (HPLC) coupled with a photodiode array (PDA) and mass spectrometry (MS) detector method. Among the tested DESs, one was selected as the best for the extraction of phenolic compounds from the hazelnut skin. For the optimization of the extraction method, a response surface methodology (RSM) was implemented, considering as independent variables the quantity of water to be added to the DES, the solid-to-solvent ratio, and the temperature of the extraction. The results, obtained using optimized conditions, were compared with those obtained with the most commonly used ethanolic UAE method.

\section{Results and Discussion}

\subsection{Screening of DESs}

The structure of DESs determines their chemical and physical properties and, consequently, influences the extraction efficiency of targeted compounds.

Eight different NADESs were tested, using choline chloride and betaine as the HBA in combination with: lactic acid (NADES-2; NADES-14), glucose (NADES-3), xylitol (NADES4), glycerol (NADES-5, NADES-11), and malic acid (NADES-10, NADES-15). Seven different DESs were tested, based on the same HBA, in combination with: urea (DES-1), 1,6-hexanediol (DES-6), triethylene glycol (DES-7, DES 13), ethylene glycol (DES-8, DES-12), and propylene glycol (DES-9) (Table 1). 
Table 1. List of the DESs and NADESs used in this study.

\begin{tabular}{cccc}
\hline Abbreviation & HBA & HBD & Molar Ratio \\
\hline DES-1 & $\mathrm{ChCl}$ & Urea & $1: 2$ \\
NADES-2 & $\mathrm{ChCl}$ & Lactic acid & $1: 2$ \\
NADES-3 & $\mathrm{ChCl}$ & Glucose & $2: 1$ \\
NADES-4 & $\mathrm{ChCl}$ & Xylitol & $1: 2$ \\
NADES-5 & $\mathrm{ChCl}$ & Glycerol & $1: 2$ \\
DES-6 & $\mathrm{ChCl}$ & 1,6-Hexanediol & $1: 7$ \\
DES-7 & $\mathrm{ChCl}$ & Triethylene glycol & $1: 2$ \\
DES-8 & $\mathrm{ChCl}$ & Ethylene glycol & $1: 2$ \\
DES-9 & $\mathrm{ChCl}$ & Propylene glycol & $1: 2$ \\
NADES-10 & $\mathrm{ChCl}$ & Malic acid & $1: 2$ \\
NADES-11 & Betaine & Glycerol & $1: 2$ \\
DES-12 & Betaine & Ethylene glycol & $1: 2$ \\
DES-13 & Betaine & Triethylene glycol & $1: 2$ \\
NADES-14 & Betaine & Lactic acid & $1: 2$ \\
NADES-15 & Betaine & Malic acid & $1: 2$ \\
\hline
\end{tabular}

The selection of DES-based extraction parameters was undertaken by determining the total phenolic compound concentration, expressed as grams of gallic acid equivalent (g GAE) per $100 \mathrm{~g}^{-1}$ of skin as a variable. In order to compare the selected DESs, all extractions were carried out under the same conditions by weighing $0.5 \mathrm{~g}$ of hazelnut skin and using a volume of $5 \mathrm{~mL}$ of extraction solvent at a temperature of $70^{\circ} \mathrm{C}$, as described in Section 3.7. Figure 1 shows the total concentration of extracted phenolic compounds with the different solvents. In general, the obtained results showed some differences in the extractability of phenolic compounds with tested DESs. No significant trend was noted in the extractions that could lead to a better performance with regard to the HBA or HBD components. The lowest amounts of total phenolic compounds were obtained using NADES-4 (7.14 g GAE $100 \mathrm{~g}^{-1}$ of skin) and NADES-11 (7.85 g GAE $100 \mathrm{~g}^{-1}$ of skin), while the highest was obtained by using DES-1, NADES-2, and DES-7, which resulted in

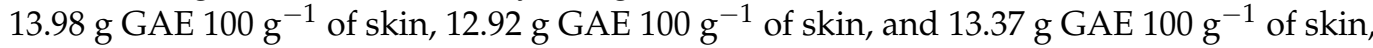
respectively. Furthermore, the ANOVA test carried out showed that there was no statistical significance among the results obtained for NADES-3, NADES-14, DES-12, or NADES-15.

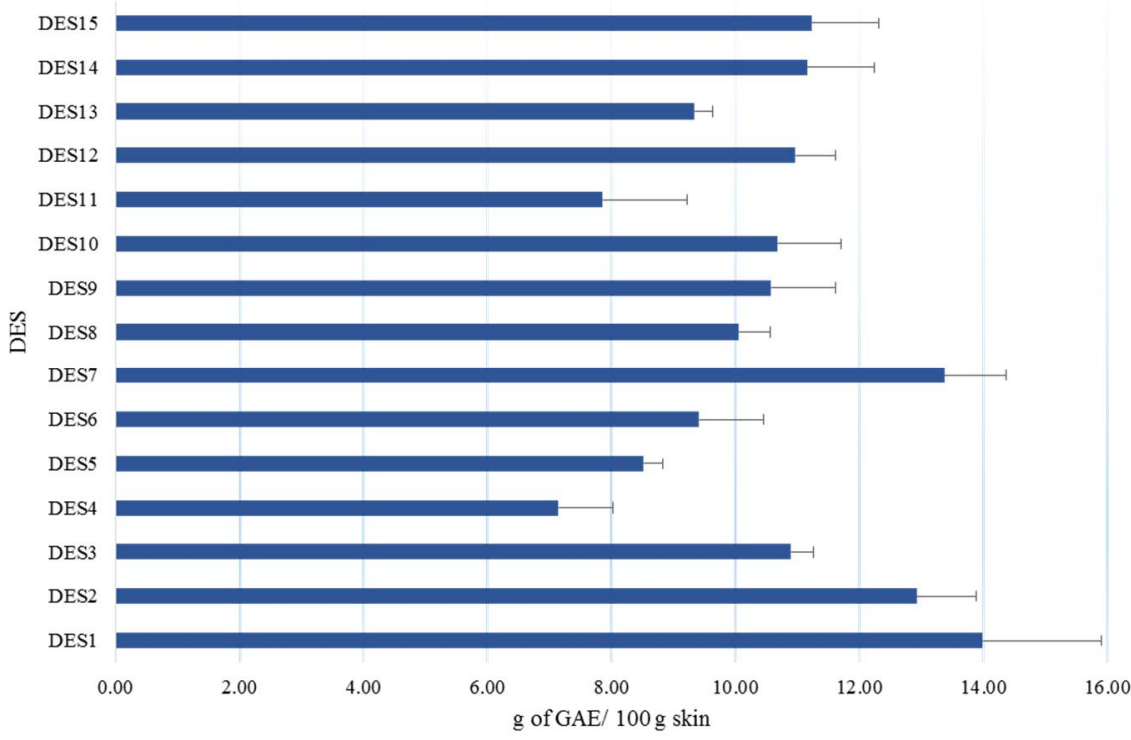

Figure 1. Effect of DES composition (Table 1) on the extraction efficiency of phenolic compounds.

Among the DESs showing the highest extraction yields, DES-1 ( $\mathrm{ChCl}$ and urea) showed an unsatisfactory repeatability, with intra- and inter-day values higher than $14 \%$, 
and for this reason it was discarded. In addition, it was also the extraction solvent that showed the worst repeatability in the extraction of phenolic compounds from olive oils [19]. Considering extraction yield and solvent toxicity, the NADES with $\mathrm{ChCl}$ and lactic acid molar ratio of 1:2 (NADES-2) was used as the extraction solvent for further optimization.

\subsection{Characterization of ChCl-Lactic Acid Molar Ratio 1:2-Based DES}

\subsubsection{FTIR Spectrum}

Figure 2 shows the spectra of the HBA, HBD, and formed NADES for the ChCl-lactic acid molar ratio of 1:2. A shift of the $\mathrm{O}-\mathrm{H}$ stretching in the lactic acid $\left(3395 \mathrm{~cm}^{-1}\right)$ and the DES $\left(3301 \mathrm{~cm}^{-1}\right)$ can be seen. In addition, a shift of the carbonilic group from $1715 \mathrm{~cm}^{-1}$ to $1730 \mathrm{~cm}^{-1}$ is present. These shifts are supposed to be present due to the hydrogen interactions between the functional groups.

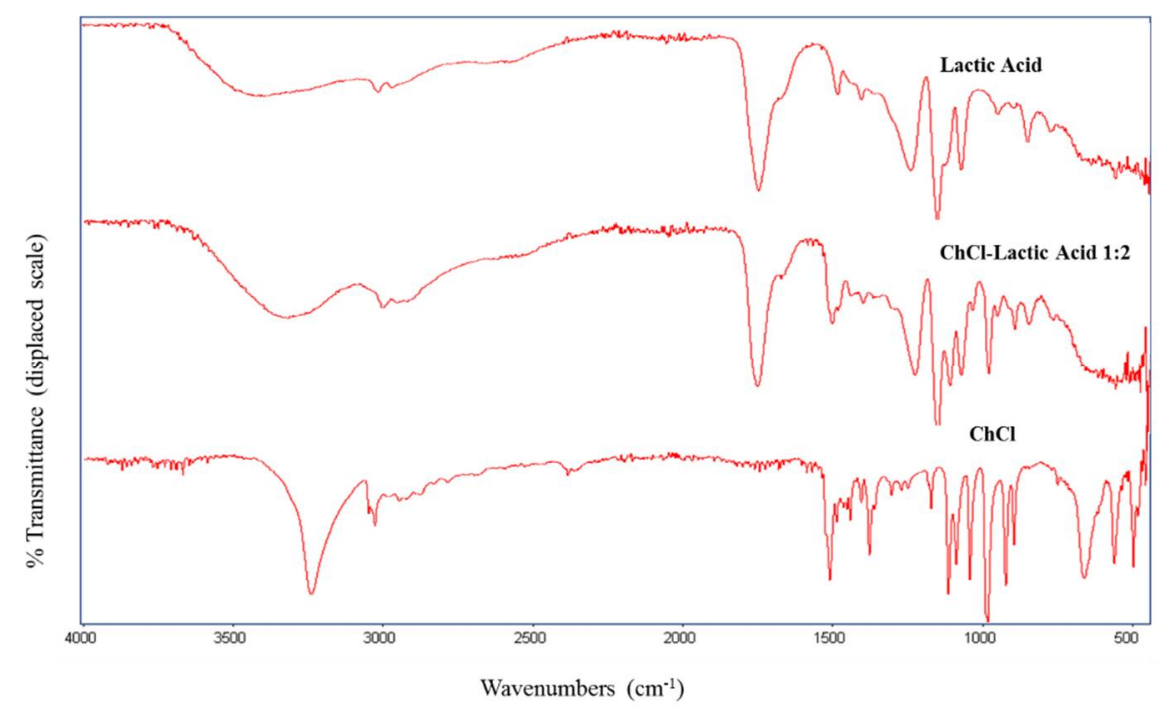

Figure 2. FTIR spectra of lactic acid, $\mathrm{ChCl}$-lactic acid molar ratio 1:2, and $\mathrm{ChCl}$.

\subsubsection{Density, $\mathrm{pH}$, and Viscosity}

Following the measurement method described in Section 3.5, the DES density showed, as expected, a trend inversely proportional to the percentage of added water, with a maximum of $1.1816 \mathrm{mg} \mathrm{mL}^{-1}$ and a minimum of $1.1146 \mathrm{mg} \mathrm{mL}^{-1}$. In general, a linear density behavior was identified for the percentage of added water; specifically, a linear decrease in the density was observed with the increase of water content in the mixture $\left(\mathrm{a}=-0.0015 ; \mathrm{b}=1.186 ; \mathrm{R}^{2}=0.9939\right)$. It is well-known that $\mathrm{pH}$ control is crucial in many of the applications of DESs. In fact, this parameter can be effective in biochemical reactions (in catalysis) and can influence the extraction yield by modulating the state of charge of the target analytes. It has been shown that the $\mathrm{pH}$ of deep eutectic solvents is influenced by the molar ratio of the component and the temperature [24,25]. pH values, measured following the procedure described in Section 3.5, ranged between 2.08 at $\mathrm{T}=24{ }^{\circ} \mathrm{C}$ and 1.2 at $83{ }^{\circ} \mathrm{C}$. The fundamental equation for the general linear model used was the following:

$$
\mathrm{pH}=\mathrm{a}+\mathrm{b} * \mathrm{~T}\left({ }^{\circ} \mathrm{C}\right)
$$

The model parameters were $\mathrm{a}=2.4492$ and $\mathrm{b}=-0.0149$, with $\mathrm{R}^{2}=0.9853$. The negative values of the slope reflect the rate of $\mathrm{pH}$ decrease with increasing temperature, as reported in the literature [24,25].

As shown in Figure 3a, the rheological behavior of the $\mathrm{ChCl}-$ lactic acid molar ratio 1:2 solvent showed the shear-thinning effect since the viscosity decreased with shear speed, at all tested temperatures. This behavior can be attributed to the breaking of the internal structure of the DES, caused both by the thermal expansion of the structure and the shear effect [26-28]. Regarding the change in the viscosity with the percentage of added water 
in the mixtures, the results shown in Figure $3 \mathrm{~b}$ confirm the extraction yield trend: the viscosity is inversely proportional to the water content. For example, at the extraction temperature of $80{ }^{\circ} \mathrm{C}$ the viscosities measured for $0 \%(v / v), 15 \%(v / v)$, and $35 \%(v / v)$ added water were $18.33 \mathrm{mPas}, 7.77 \mathrm{mPas}$, and $3.01 \mathrm{mPas}$, respectively. This was probably related to the better mixing between the matrix and the extraction solvent that occurs when the water content in the DES is higher. Moreover, we noticed that the percentage decrease of viscosity with increasing temperature (from $20^{\circ} \mathrm{C}$ to $80^{\circ} \mathrm{C}$ ) was around $90 \%$ for all the tested water percentages.

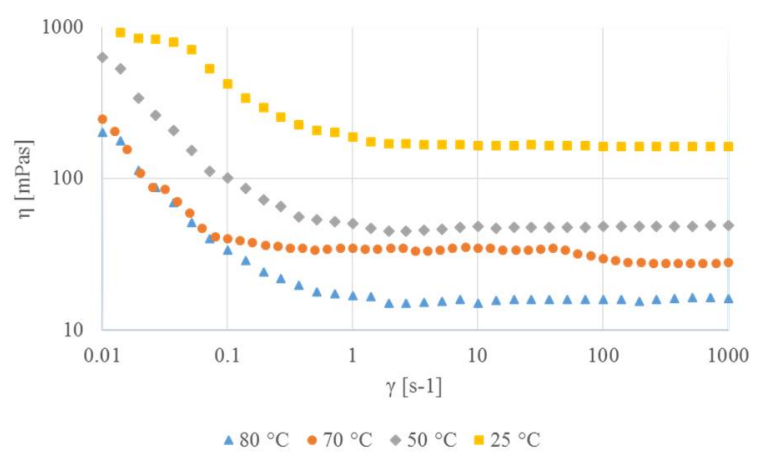

(a)

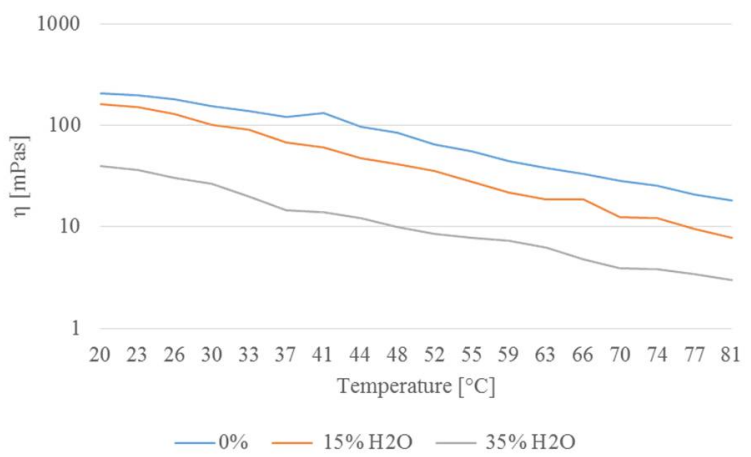

(b)

Figure 3. (a) Viscosity $(\eta)$ versus shear rate $(\gamma)$ change at different temperatures (blue $80{ }^{\circ} \mathrm{C}$, orange $70{ }^{\circ} \mathrm{C}$, grey $50{ }^{\circ} \mathrm{C}$, yellow $\left.25^{\circ} \mathrm{C}\right)$; (b) viscosity $(\eta)$ versus temperature change for different water contents $(\% v / v)$ at $g=1\left[\mathrm{~s}^{-1}\right]$.

\subsection{Selection of Extraction Parameters and Response Surface Methodology Optimization}

The response surface methodology (RSM) optimization was conducted with the aim of determining the optimal experimental conditions for the total phenolic compounds extracted. Based on our preliminary data and the currently available literature, we selected the following independent variables for the RSM in this study: water content in the DES (15 and $35 \%)$; extraction temperature $\left(50,70\right.$, and $\left.80^{\circ} \mathrm{C}\right)$; and solid-to-solvent ratio (1:3.33, 1:5, 1:6.25, 1:10, and 1:25 gmL ${ }^{-1}$ ).

Maximum temperature was set at $80{ }^{\circ} \mathrm{C}$ in order to avoid partial degradation of extracted compounds [29] while maximum percentage of water added was set as $35 \%$. Studies from the literature confirm that the use of different percentages $(v / v)$ of water in DESs at can be exploited to modulate certain chemical and physical characteristics [30].

Data from previous studies has shown that DES/NADES water systems are stable up to an added water content of around 50\% ( $v / v)$ [31]. In addition, Lanjekar et al. used a NADES consisting of ChCl-lactic acid mixed with up to $30 \%$ water for the extraction of glycyrrhizic acid from Glycyrrhiza glabra, thus demonstrating its stability, which was confirmed by the unchanged -OH stretching signal between the DES water system and the DES without the addition of water [32]. Higher values are known to cause DES solubilization by breaking up the hydrogen interactions among the components and promoting hydrogen interactions between the water molecules themselves [33]. A previous study showed that a water addition greater than $30 \%$ leads to a decrease of genistin, genistein, and apigenin extraction yield from pigeon pea roots, since excessive water can decrease the hydrogen bond strength between the DES and the target compounds [34].

The experimental data acquired in a randomized order (Table 2) were fitted into a second-order polynomial equation (Equation (2)), described in Section 3.8 with the coefficients listed in Table 3. System behavior was represented using three-dimensional response surface plots. Every surface was evaluated by fixing one value for an independent variable and varying the others according to their experimental domains. The total phenolic compounds in the extracts obtained varied from 6.14 (g GAE) $100 \mathrm{~g}^{-1}$ of skin to 16.95 (g GAE) $100 \mathrm{~g}^{-1}$ of skin, depending on the different levels of the investigated parameters. 
The lowest extraction yield for all investigated responses was obtained using $15 \%(v / v)$ water, with a temperature of $50{ }^{\circ} \mathrm{C}$ and a solid-to-solvent ratio of 1:3.33. Figure 3 shows the response surface plots for the total phenolic compounds concentration for each pair of independent factors.

Table 2. Dataset of the experiment.

\begin{tabular}{|c|c|c|c|c|c|}
\hline Standard Order ${ }^{a}$ & Run Order ${ }^{b}$ & $\mathbf{T}^{\circ} \mathrm{C} \pm \mathbf{2}$ & Time (min) & $\% \mathrm{H}_{2} \mathrm{O}(v / v)$ & g skin $/ 5 \mathrm{~mL}$ \\
\hline 1 & 9 & 50 & 30 & 15 & 0.2 \\
\hline 2 & 23 & 50 & 30 & 15 & 0.5 \\
\hline 3 & 19 & 50 & 30 & 15 & 0.8 \\
\hline 4 & 3 & 50 & 30 & 15 & 1 \\
\hline 5 & 26 & 50 & 30 & 15 & 1.5 \\
\hline 6 & 7 & 50 & 30 & 35 & 0.2 \\
\hline 7 & 13 & 50 & 30 & 35 & 0.5 \\
\hline 8 & 17 & 50 & 30 & 35 & 0.8 \\
\hline 9 & 5 & 50 & 30 & 35 & 1 \\
\hline 10 & 29 & 50 & 30 & 35 & 1.5 \\
\hline 11 & 14 & 70 & 30 & 15 & 0.2 \\
\hline 12 & 8 & 70 & 30 & 15 & 0.5 \\
\hline 13 & 15 & 70 & 30 & 15 & 0.8 \\
\hline 14 & 22 & 70 & 30 & 15 & 1 \\
\hline 15 & 28 & 70 & 30 & 15 & 1.5 \\
\hline 16 & 25 & 70 & 30 & 35 & 0.2 \\
\hline 17 & 10 & 70 & 30 & 35 & 0.5 \\
\hline 18 & 1 & 70 & 30 & 35 & 0.8 \\
\hline 19 & 20 & 70 & 30 & 35 & 1 \\
\hline 35 & 12 & 70 & 30 & 35 & 1.5 \\
\hline 21 & 11 & 80 & 30 & 15 & 0.2 \\
\hline 22 & 24 & 80 & 30 & 15 & 0.5 \\
\hline 23 & 18 & 80 & 30 & 15 & 0.8 \\
\hline 24 & 6 & 80 & 30 & 15 & 1 \\
\hline 25 & 16 & 80 & 30 & 15 & 1.5 \\
\hline 26 & 27 & 80 & 30 & 35 & 0.2 \\
\hline 27 & 4 & 80 & 30 & 35 & 0.5 \\
\hline 28 & 21 & 80 & 30 & 35 & 0.8 \\
\hline 29 & 30 & 80 & 30 & 35 & 1 \\
\hline 30 & 2 & 80 & 30 & 35 & 1.5 \\
\hline
\end{tabular}

a Not randomized; ${ }^{\text {randomized. }}$

Table 3. Regression coefficients of predicted quadratic polynomial model.

\begin{tabular}{ccccccccccc}
\hline & \multicolumn{3}{c}{ Quadratic } & \multicolumn{3}{c}{ Crossproduct } & \multicolumn{3}{c}{ Linear } \\
\hline \multirow{2}{*}{ Coefficient } & $\mathrm{a}_{1}$ & $\mathrm{a}_{2}$ & $\mathrm{a}_{3}$ & $\mathrm{a}_{4}$ & $\mathrm{a}_{5}$ & $\mathrm{a}_{6}$ & $\mathrm{a}_{7}$ & $\mathrm{a}_{8}$ & $\mathrm{a}_{9}$ & $\mathrm{a}_{10}$ \\
& 0.6394 & 0 & 2.8390 & 0.1928 & -0.9318 & -0.4731 & 0.7166 & 1.0958 & -3.3169 & 6.7465 \\
\hline
\end{tabular}

Figure $4 \mathrm{a}$ shows the system response to water content and solid-to-solvent ratio variations at a constant temperature of $80^{\circ} \mathrm{C}$. The increase in response is related to the increase in water percentage and solid-to-solvent ratio. The extraction yield variation between the minimum water content $(15 \% v / v)$ and the maximum one $(35 \% v / v)$ fixed the solid-to-solvent ratio at $1: 25 \mathrm{gmL}^{-1}$, which is about $3 \%$. Figure $4 \mathrm{~b}$ shows the dependence of the extraction yield on the temperature and the solid-to-solvent ratio, keeping a constant water content of $35 \%(v / v)$. As shown, a higher temperature and higher solid-to-solvent ratio favored extraction. The variation of the extraction yield in response to temperature changes (from $50{ }^{\circ} \mathrm{C}$ to $80^{\circ} \mathrm{C}$ ), at the optimum solid-to-solvent ratio $\left(1: 25 \mathrm{gmL}^{-1}\right)$, was about $4.5 \%$. In Figure $4 a, b$, an increase of $9.44 \%$ in extraction yield can be highlighted when varying the solid-to-solvent ratio between the minimum $\left(1: 3.33 \mathrm{gmL}^{-1}\right)$ and maximum $(1: 25$ $\mathrm{gmL}^{-1}$ ) values. Figure $4 \mathrm{c}$ shows the extraction yield trend in response to varying water 
percentage and temperature and a fixed solid-to-solvent ratio of $1: 25 \mathrm{gmL}^{-1}$. It is clear that higher values for both water content and temperature lead to a better extraction yield.

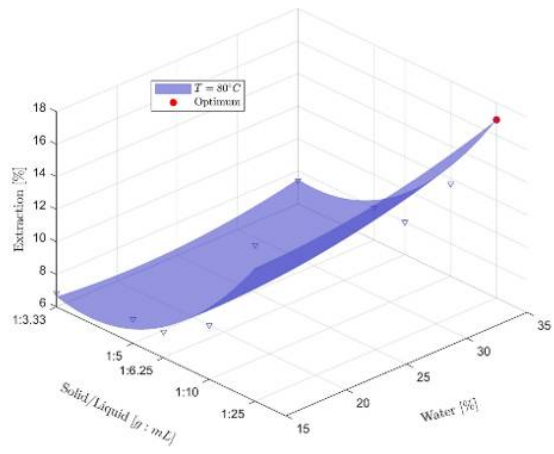

(a)

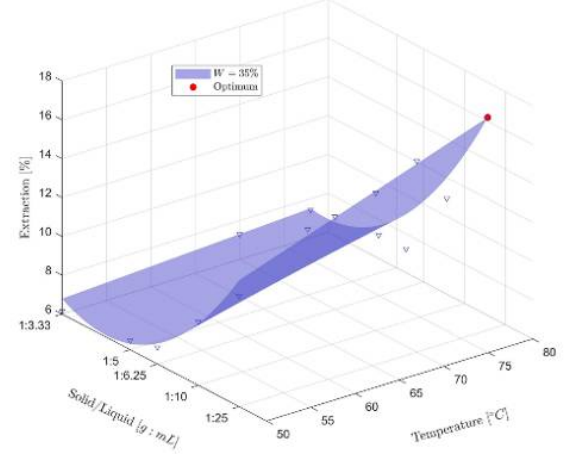

(b)

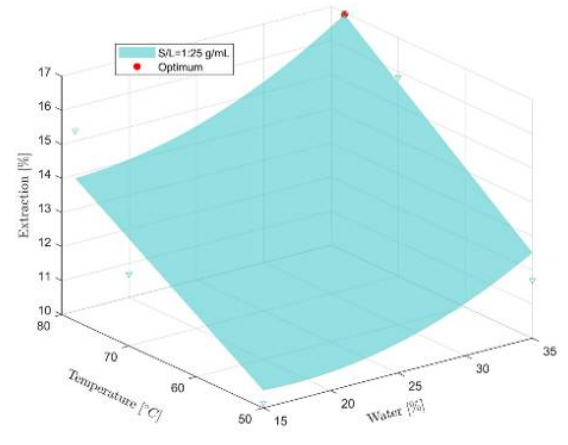

(c)

Figure 4. Three-dimensional plots of response surfaces for the effects on the response of (a) the solid-to-liquid ratio and percentage of water (g GAE $100 \mathrm{~g}^{-1}$ of skin) at three different temperatures; (b) the solid-to-liquid ratio and temperature ( $\mathrm{g}$ GAE $100 \mathrm{~g}^{-1}$ of skin) at two different percentages of water; (c) the temperature and percentage of water ( $\mathrm{g}$ GAE $100 \mathrm{~g}^{-1}$ of skin) at five different solid-to-liquid ratios.

To evaluate the correlation between the predicted values of the extraction yield obtained from the polynomial equation and the experimental ones, experimental and predicted values are plotted together in Figure 5. The determination coefficient was calculated as $\mathrm{R}^{2}=0.92$, indicating a good correlation between predicted and experimental data. The

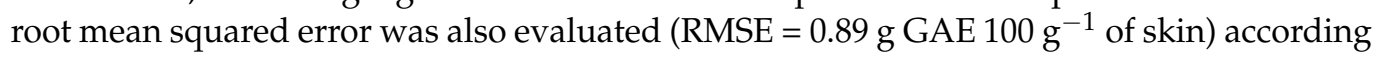
to the presented model regression parameters. The optimal predicted point was found at the following conditions: $\mathrm{T}=80^{\circ} \mathrm{C}, \mathrm{W}=35 \%$, and $\mathrm{SL}=1: 25 \mathrm{gmL}^{-1}$, which correspond to a maximum predicted extraction yield of $16.96 \mathrm{~g} \mathrm{GAE} 100 \mathrm{~g}^{-1}$ of skin. An additional experimental test was conducted setting $\mathrm{T}, \mathrm{W}$, and SL as equal to the optimal model estimation. The extraction yield obtained was $16.80 \pm 0.09 \mathrm{~g}$ GAE $100 \mathrm{~g}^{-1}$ of skin, thus confirming the data.

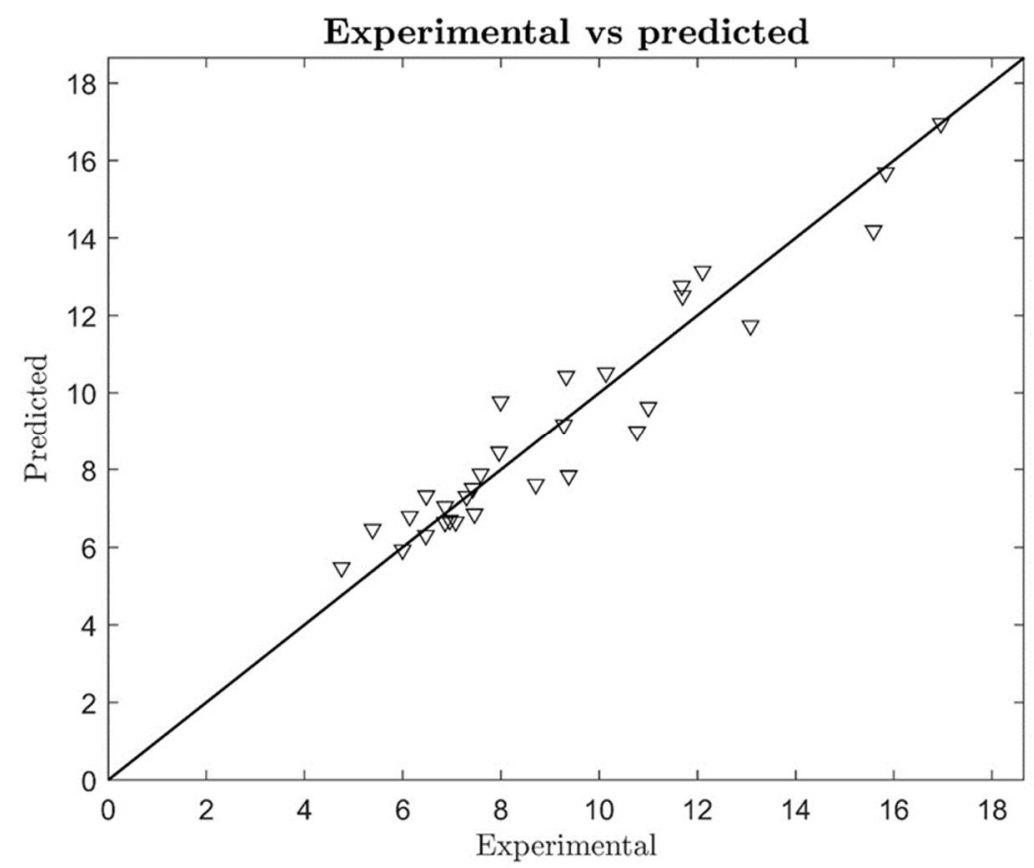

Figure 5. Residues. 
In order to compare the DES-based extraction with a conventional one, an organic solvent-based UAE was conducted using $0.5 \mathrm{~g}$ of skin extracted with $5 \mathrm{~mL}$ of EtOH- $\mathrm{H}_{2} \mathrm{O}$ $50 \%(v / v)$ mixtures at room temperature for $30 \mathrm{~min}$. The total concentration of extracted phenolic compounds was $10.22 \mathrm{~g}$ GAE $100 \mathrm{~g}^{-1}$ of skin. Under optimal conditions, the DES-based system makes it possible to extract in a single step 39\% more than the amount extracted by a single-step extraction with $\mathrm{EtOH}-\mathrm{H}_{2} \mathrm{O} 50 \%(v / v)$ solvent.

\subsection{HPLC-PDA/MS Analysis of Hazelnut Skin Extract}

Figure 6 shows the HPLC profile, at 280 and $360 \mathrm{~nm}$, of the phenolic compounds extracted using the conventional solvent identified. Table 4 reports the phenolic compounds tentatively identified, including the experimental $\mathrm{m} / \mathrm{z}$ values. As shown in Table 4 , the identified phenolic compounds were flavan-3-ols and phenolic acids.

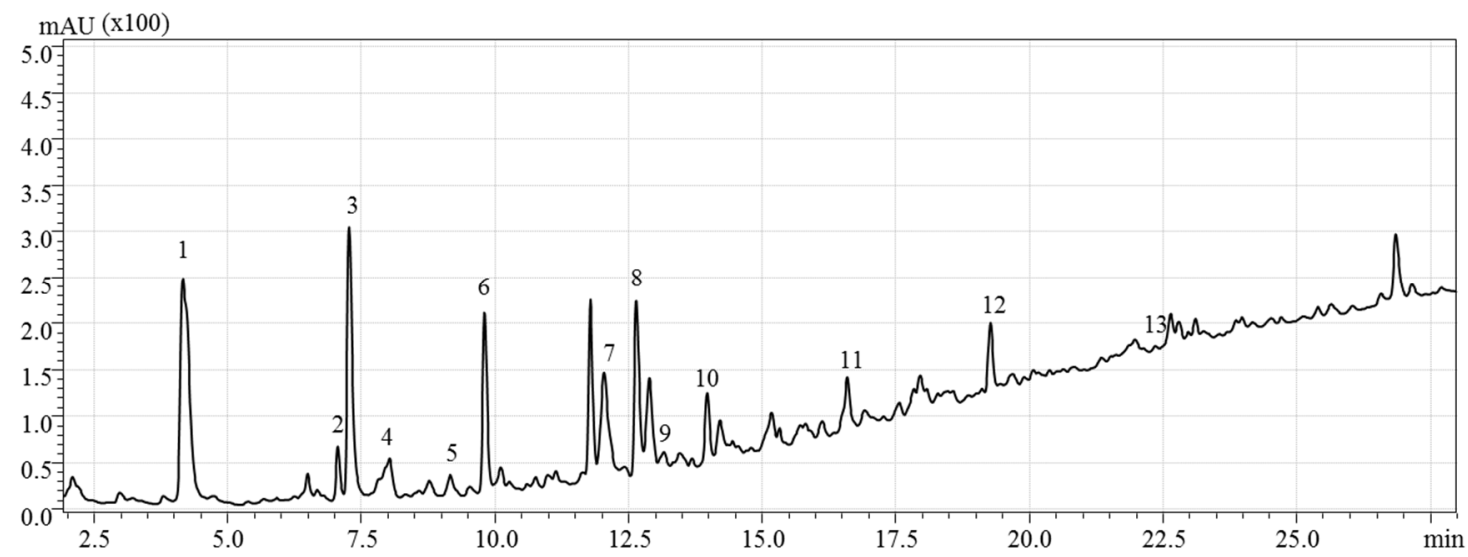

(a)

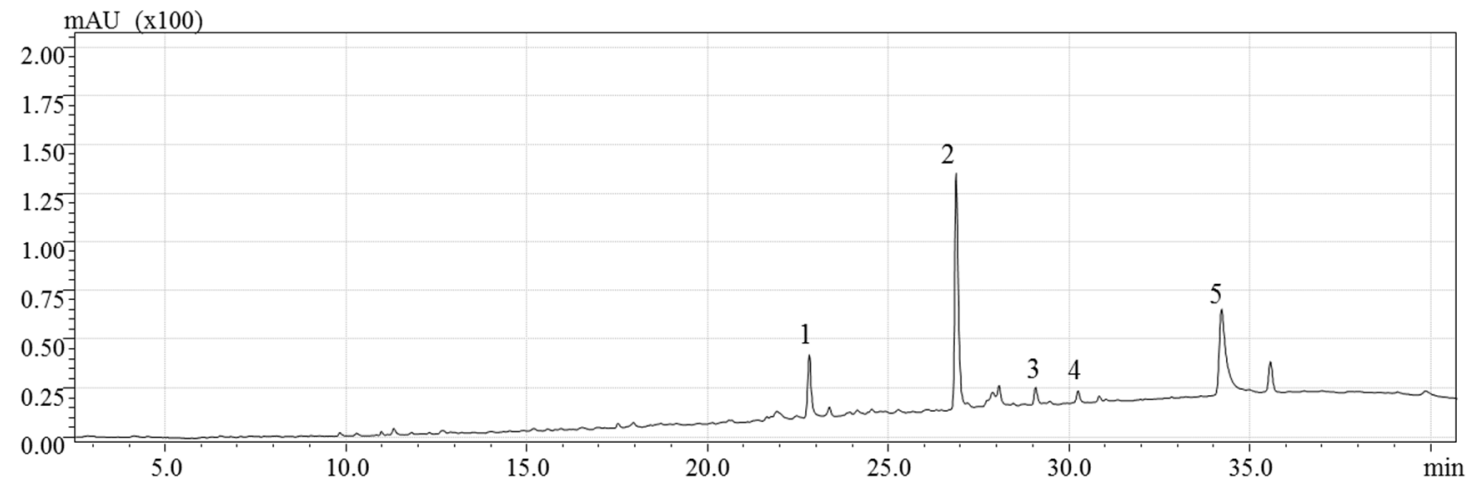

(b)

Figure 6. HPLC-PDA chromatogram of hazelnut skin phenolic compounds extract. (a) Compounds detected at $280 \mathrm{~nm}: 1$, gallic acid; 2, protocatechuic acid; 3, procyanidin trimer $\mathrm{C}$; 4, prodelphinidin beta-type dimer; 5 , prodelphinidin beta-type dimer; 6 , prodelphinidin beta-type dimer; 7 , procyanidin beta 1 dimer; $8,(+)$ catechin; 9 , procyanidin beta-type trimer; 10, procianidin beta-type trimer; 11 (-) epicatechin; 12, procyanidin beta-type dimer gallate; 13, epicatechin 3-O-gallate. (b) Compounds detected at $360 \mathrm{~nm}$ : 1, myricetin rhamnoside; 2, quercetin-3-O-rhamnoside; 3, ploretin-2-O-glucoside; 4, kampferol rhamnoside; 5 , quercetin. 
Table 4. Chromatographic data regarding the phenolic compounds identified in hazelnut skin extract by HPLC-PDA/ESI-MS (280 $\mathrm{nm}$ and $360 \mathrm{~nm}$ ).

\begin{tabular}{|c|c|c|}
\hline \multicolumn{3}{|c|}{$\lambda 280 \mathrm{~nm}$} \\
\hline Number & Compound & {$[\mathbf{M}-\mathbf{H}]^{-}(m / z)$} \\
\hline 1 & Gallic acid & 169 \\
\hline 2 & Protocatechuic acid & 153 \\
\hline 3 & Procyanidin trimer C2 & 865 \\
\hline 4 & Prodelphinidin beta-type dimer & 593 \\
\hline 5 & Prodelphinidin beta-type dimer & 593 \\
\hline 6 & Prodelphinidin beta-type dimer & 593 \\
\hline 7 & Procyanidin beta 1 dimer & 577 \\
\hline 8 & $(+)$ Catechin & 289 \\
\hline 9 & Procyanidin beta-type trimer & 865 \\
\hline 10 & Procyanidin beta-type trimer & 865 \\
\hline 11 & $(-)$ Epicatechin & 289 \\
\hline 12 & Procyanidin beta-type dimer gallate & 729 \\
\hline 13 & Epicatechin 3-O-gallate & 441 \\
\hline \multicolumn{3}{|c|}{$\lambda 360 \mathrm{~nm}$} \\
\hline Number & Compound & {$[\mathbf{M}-\mathbf{H}]^{-}(m / z)$} \\
\hline 1 & Myricetin rhamnoside & 463 \\
\hline 2 & Quercetin-3-O-rhamnoside & 447 \\
\hline 3 & Ploretin-2-O-glucoside & 435 \\
\hline 4 & Kampferol rhamnoside & 431 \\
\hline 5 & Quercetin & 301 \\
\hline
\end{tabular}

The putative identification of the 18 detected compounds was based on the massto-charge ratio $(\mathrm{m} / \mathrm{z})$ of the molecular ion, the retention time, and UV data from the literature [1]. Whenever possible, analytical standards were used to confirm both the retention times and the mass-to-charge ratios of the compounds.

Flavan-3-ols (+)-catechin and (-)-epicatechin were distinguished by their different retention times (10.2 $\mathrm{min}$ and $14 \mathrm{~min}$, respectively), since they have the same molecular ion $\mathrm{m} / \mathrm{z}$ ratio $(289 \mathrm{~m} / \mathrm{z})$. These flavan-3-ols were detected in all the hazelnut components (i.e., shell, kernel, and skin) $[1,35,36]$. Epicatechin 3-O-gallate was detected with $[\mathrm{M}-$ $\mathrm{H}]^{-}$at $m / z$ 441, confirming the results obtained by Del Rio et al. (2011) [1]. Among proanthocyanidins, one B-type procyanidin (PC) dimer was identified ([M $-\mathrm{H}]^{-}$at $m / z$ 577). Three procyanidin trimers were detected with a mass-to-charge ratio of $865 \mathrm{~m} / \mathrm{z}$. Based on the elution time, which precedes that of procyanidin B1, one was tentatively identified as procyanidin trimer $\mathrm{C} 2$, while the others were identified as procyanidin betatype trimers. Within the phenolics acids, protocatechuic acid $\left([\mathrm{M}-\mathrm{H}]^{-}\right.$at $\left.153 \mathrm{~m} / z\right)$ and gallic acid $\left([\mathrm{M}-\mathrm{H}]^{-}\right.$at $\left.169 \mathrm{~m} / z\right)$ were also identified through comparison with analytical standards and in accordance with data from previous studies (Del Rio et al., 2011 [1], Slatnar et al., 2014 [36], and Yuan et al., 2018 [37]). Three flavonol rhamnoside compounds were identified, these being myricetin rhamnoside $\left([\mathrm{M}-\mathrm{H}]^{-}\right.$at $463 \mathrm{~m} / \mathrm{z}$ ), quercetin 3-O-rhamnoside ([M - H $]^{-}$at $\left.447 \mathrm{~m} / z\right)$, and kaempferol rhamnoside $\left([\mathrm{M}-\mathrm{H}]^{-}\right.$ at $431 \mathrm{~m} / z$ ). The same compounds have been previously detected in hazelnut skin [1] and, with the exception of kaempferol rhamnoside, they have also been previously identified in leaves, shells, and kernels of C. avellana L. [35-37]. Quercetin ([M - H] $]^{-}$at $\left.301 \mathrm{~m} / z\right)$ was also detected by comparison with analytical standards. Moreover, this aglycon has been previously identified in hazelnut skin and shell $[1,37]$. Finally, phloretin 2-O-glucoside ([M $-\mathrm{H}]^{-}$at $m / z 435$ ), belonging to the dihydrochalcone subclass, was also identified. 


\section{Materials and Methods}

\subsection{Chemicals}

$\mathrm{ChCl}$, triethylene glycol, glycerol, urea, and sucrose were obtained from Sigma-Aldrich (Milan, Italy); sorbitol and glucose were purchased from Fisher Scientific Italia (Milan, Italy); and betaine anhydrous, xylitol, citric acid, ethylene glycol, propylene glycol, 1,6hexanediol, lactic acid, and malic acid were purchased from Carlo Erba (Milan, Italy). Gallic acid, procyanidin B2 (PB2), procyanidin A2 (PA2), epicatechin, quercetin, kaempferol, methanol (MeOH) (purity 99.9\%), water (HPLC-MS grade), acetonitrile (purity 99.9\%), and formic acid (purity 95-97\%) were obtained from Sigma-Aldrich (Milan, Italy). All the chemicals were of analytical reagent grade and were used without any further purification.

\subsection{Samples}

Hazelnut skins were obtained from a company that uses hazelnuts as a raw material for the preparation of food products. Before the experiments, an aliquot of sample was ground in a coffee grinder (Moulinex Super Junior) and stored at room temperature until use.

\subsection{Preparation of DESs}

Due to its hygroscopicity, $\mathrm{ChCl}$ was dried in an oven for one night at $90{ }^{\circ} \mathrm{C}$ before preparing the DESs. HBA and HBD were weighed in the established molar ratio and placed in a round-bottom flask. The mixture was heated at $80{ }^{\circ} \mathrm{C}$ until a homogeneous liquid was formed. Then, water was added $(35 \%(v / v))$ in order to reduce the viscosity of the DESs. All the selected DESs are summarized in Table 1.

\subsection{FTIR Spectroscopy}

DESs were analyzed by FTIR analysis with a Nicolet 8700 to ensure the formation of the DES. The spectra were acquired at room temperature with six replicates (64 scans for acquisition); the wavenumber measurement range was from 4000 to $400 \mathrm{~cm}^{-1}$ with a $4 \mathrm{~cm}^{-1}$ resolution.

\section{5. $p H$, Density, and Viscosity of DESs}

A $0.5 \mathrm{M}$ aqueous solution of DES was prepared for $\mathrm{pH}$ measurements and kept at room temperature until use, following Skulcova et al., 2018 [38]. The $\mathrm{pH}$ was measured at different temperatures in a range from $25{ }^{\circ} \mathrm{C}$ to $80^{\circ} \mathrm{C}$ by heating the mixture in a water bath on a heating plate. $\mathrm{pH}$ was determined using a digital $\mathrm{pH}$ meter from Eutech Instruments $^{\circledR}$ (Toronto, ON, Canada). DES density was measured by weighing a $1 \mathrm{~mL}$ flask filled with the DES on an analytical balance from Sartorious (Gottinga, Germany) at room temperature. The dynamic viscosity $(\eta)$ was measured with an Anton Paar rheometer MCR 302 (Anton Paar, Austria) in a shear rate range between 0.01 and $1000 \mathrm{~s}^{-1}$, with a parallelplate measurement geometry with a gap of $0.100 \mathrm{~mm}$. The viscosity was investigated across a range of temperatures $\left(25^{\circ} \mathrm{C}\right.$ to $\left.80^{\circ} \mathrm{C}\right)$ and for three different percentages of water in the DES $(0 \%, 15 \%$, and $35 \%)$ in order to correlate these characteristics with the extraction efficiency. All the experimental measurements were carried out in triplicate and the mean values were used.

\subsection{Ultrasound-Assisted Extraction of Phenolic Compounds with a Conventional Solvent}

In order to compare the efficiency of DES-based extraction with a conventional solventbased one, a method from the literature was chosen [1], with some modifications. First, $0.5 \mathrm{~g}$ of sample was extracted using $5 \mathrm{~mL}$ of a mixture of $\mathrm{EtOH}$ and $\mathrm{H}_{2} \mathrm{O}(50: 50, v / v)$ in a centrifuge tube, and the mixture was placed in an ultrasound bath (Elmasonic $\mathrm{S} 30 \mathrm{H}$, Elma Schmidbauer $\mathrm{GmbH}$, Singen, Germany) at $50{ }^{\circ} \mathrm{C}$, frequency of $37 \mathrm{kHz}$ and heating power of $200 \mathrm{~W}$, for $60 \mathrm{~min}$. Then, the tube was centrifuged at $3214 \mathrm{~g}$ for $5 \mathrm{~min}$ and the supernatant was filtered in a $0.45 \mu \mathrm{m}$ syringe filter. The solution was dried under vacuum and reconstituted with $400 \mu \mathrm{L}$ of $\mathrm{MeOH}-\mathrm{H}_{2} \mathrm{O}$ 50:50 (v/v) mixture. Each extraction was conducted in triplicate and all the extracts were stored at $-80^{\circ} \mathrm{C}$ until use. 


\subsection{Ultrasound-Assisted Extraction of Phenolic Compounds with DESs}

An total of $0.2 \mathrm{~g}$ of hazelnut skin was mixed with $5 \mathrm{~mL}$ of DES and vortexed for $1 \mathrm{~min}$. The tube was put in an ultrasound bath (Elmasonic S30H, Elma Schmidbauer $\mathrm{GmbH}$, Singen, Germany), frequency of $37 \mathrm{kHz}$ and heating power of $200 \mathrm{~W}$, for $30 \mathrm{~min}$ at a temperature of $80^{\circ} \mathrm{C}$ with a temperature control of $\pm 2{ }^{\circ} \mathrm{C}$. The mixture was filtered on a Buchner filter and the extract was stored at $-80^{\circ} \mathrm{C}$ until analysis. Triplicate extractions were conducted for all experiments.

\subsection{Ultrasound-Assisted Extraction of Phenolic Compounds with DESs}

A selection process was performed to select the best experimental conditions for extraction of phenolic compounds. Factors such as water content in the DES, extraction temperature, and the solid-to-solvent ratio were studied.

Two levels were selected for the water content added to the DES mixture $(15 \%$ and $35 \%, v / v)$, five levels for the solid-to-solvent ratio $\left(\mathrm{gmL}^{-1}\right), 1: 3.33,1: 5,1: 6.25,1: 10$, and 1:25, and three levels for the extraction temperature $\left(50^{\circ} \mathrm{C}, 70^{\circ} \mathrm{C}\right.$, and $\left.80^{\circ} \mathrm{C}\right)$. For the evaluation of extraction time, preliminary studies showed that after $30 \mathrm{~min}$ there was no significant variation in the extracted amount (data not shown) and thus this time period was selected as extraction time. The experiments, conducted by simply matching each of the levels of the factors to each other, are summarized in Table 2. Experimental data were fitted into a second-order polynomial model and regression coefficients were obtained. The polynomial model formula was as follow:

$$
y=a_{1} W^{2}+a_{2} T^{2}+a_{3} S^{2}+a_{4} W T+a_{5} T S L+a_{6} W S L+a_{7} W+a_{8} T+a_{9} S L+a_{10}
$$

where $\mathrm{a}_{1-10}$ are the coefficients of the polynomial model equation obtained by means of a nonlinear regression, while $\mathrm{W}$ (water content, $\% v / v$ ), T (temperature, ${ }^{\circ} \mathrm{C}$ ), and SL (solid-to-solvent ratio, gmL ${ }^{-1}$ ) represent the independent coded variables and $\mathrm{y}$, the model output, is the extracted amount in $\mathrm{g} \mathrm{GAE} 100 \mathrm{~g}^{-1}$ of skin. Each variable was mapped linearly in the interval $[-1 ; 1]$, where -1 corresponded to the variable minimum value and 1 to the variable maximum value. MATLAB ${ }^{\circledR}$ was used as the software for the regression and the response surfaces analysis and visualization. The analysis consisted of evaluating the absolute maximum of the curve, which corresponded to the optimal operating conditions confirmed experimentally.

\subsection{HPLC-PDA/ESI-MS Analysis of Phenolic Compounds}

The analysis was performed using a Shimadzu Prominence LC-20A instrument (Shimadzu, Milan, Italy) equipped with two LC-20 AD XR pumps-a SIL-10ADvp pump and a CTO-20 AC column oven-and a DGU-20 A3 degasser coupled to a SPD-M10Avp PDA detector and a mass spectrometer detector (LCMS-2010, Shimadzu, Tokyo, Japan), equipped with an electrospray (ESI) interface. Shimadzu LCsolution version 3.7 software (Shimadzu, version 3.7) was used to acquire the MS data. The separation of the analytes was carried out using a Core Shell C18 column $(150 \times 4.6 \mathrm{~mm}$ I.D., $2.7 \mu \mathrm{m}$ d.p. $)$ (Merck KGaA, Darmstadt, Germany). Elution was performed at a constant flow rate of $1 \mathrm{mLmin}^{-1}$ and at a temperature of $40^{\circ} \mathrm{C}$. The mobile phase was (A) $0.1 \%(v / v) \mathrm{HCOOH}$ aqueous solution and (B) acetonitrile with $0.1 \%(v / v) \mathrm{HCOOH}$. Polyphenols were separated using the following gradients: $0-40 \mathrm{~min} 0-30 \% \mathrm{~B}, 40-41 \mathrm{~min} 100 \% \mathrm{~B}$. The injection volume was $2 \mu \mathrm{L}$. Data were acquired using aPDA in the range $200-400 \mathrm{~nm}$ and the chromatograms were extracted at 280 and $360 \mathrm{~nm}$. MS chromatograms were acquired in negative ionization mode, using the following parameters: nebulizing gas flow rate $\left(\mathrm{N}_{2}\right): 1.5 \mathrm{~mL} \mathrm{~min}^{-1}$; event time: $1 \mathrm{~s}$; mass spectral range: $\mathrm{m} / z$ 100-800; scan speed: $1000 \mathrm{amu} / \mathrm{s}$; detector voltage: $1.5 \mathrm{kV}$; interface temperature: $250{ }^{\circ} \mathrm{C}$; CDL temperature: $300{ }^{\circ} \mathrm{C}$; heat block temperature: $300{ }^{\circ} \mathrm{C}$; interface voltage: $-3.50 \mathrm{kV}$; Q-array voltage: $0.0 \mathrm{~V}$; Q-array RF: $150.0 \mathrm{~V}$. 


\subsection{Determination of Total Phenolic Compounds Content}

The total phenolic compounds (TPCs) content was determined by oxidation of phenolic compounds using Folin-Ciocalteu's reagent [39]. Briefly, $20 \mu \mathrm{L}$ of sample were mixed with $100 \mu \mathrm{L}$ of Folin-Ciocalteu's reagent and $1580 \mu \mathrm{L}$ of EtOH: $\mathrm{H}_{2} \mathrm{O}(50 \% v / v)$ mixture and kept in the dark for $10 \mathrm{~min}$. Then, $300 \mu \mathrm{L}$ of an aqueous solution of $\mathrm{Na}_{2} \mathrm{CO}_{3} 0.2 \mathrm{~g} \mathrm{~mL}^{-1}$ was added and put back in the dark for $2 \mathrm{~h}$ under continuous stirring. Finally, the mixture was centrifuged for $2 \mathrm{~min}$ at 10,621 g and $200 \mu \mathrm{L}$ of the sample was put in a Greiner microplate. The absorbance was measured with the Infinite M200 PRO Tecan microplate spectrophotometer (Tecan Trading AG, Switzerland) at $765 \mathrm{~nm}$. The concentration of the samples was calculated by interpolating the result in a calibration curve made using gallic acid as an analytical standard in a range from 0 to $2000 \mu \mathrm{g} \mathrm{mL}^{-1}$. Results were expressed as grams of gallic acid equivalent ( $\mathrm{g} \mathrm{GAE}$ ) per $100 \mathrm{~g}^{-1}$ of skin.

\section{Conclusions}

The extraction of bioactive compounds such as flavan-3-ols and phenolic acids from hazelnut skin was studied with deep eutectic solvents. HPLC-PDA/MS analysis revealed the presence of 18 compounds in the samples. Among the DESs tested, the solvent composed of $\mathrm{ChCl}$ as the HBA and lactic acid as the HBD (NADES-2) was selected as the most promising neoteric solvent. This eutectic solvent was successfully employed in a simple and fast extraction procedure supported by UA and optimized with the response surface methodology. The individual optimized factors can be classified according to their contribution to maximizing extraction yield as follows: solid-to-solvent ratio, temperature, and percentage of added water in the DES. Under optimal conditions, this system makes it possible to extract $39 \%$ more than the amount extracted with organic solvent-based extraction. Overall, this work contributes to the possibility of developing an efficient and rapid extraction process to recover natural antioxidants from hazelnut byproducts using bio-renewable DESs, as an opportunity to stabilize new food waste recovery strategies based on sustainable solvents.

Author Contributions: Conceptualization, C.F., V.P. and L.D.G.; Data curation, V.G.; Formal analysis, L.M. and M.C.; Investigation, C.F. and V.G.; Methodology, S.D.P.; Project administration, L.D.G.; Resources, L.D.G.; Supervision, C.F.; Visualization, V.G. and L.D.; Writing-original draft, C.F. and V.G.; Writing-review \& editing, S.D.P., L.D., L.M., M.C., V.P. and L.D.G. All authors have read and agreed to the published version of the manuscript.

Funding: This research was funded by Soremartec Italia Srl (Alba, Cuneo, Italy).

Institutional Review Board Statement: Not applicable.

Informed Consent Statement: Not applicable.

Data Availability Statement: Not applicable.

Acknowledgments: We would like to thank Soremartec Italia Srl (Alba, Cuneo, Italy) for their support.

Conflicts of Interest: The authors declare no conflict of interest.

Sample Availability: Samples of the compounds extracted from hazelnut skin are available from the authors.

\section{References}

1. Del Rio, D.; Calani, L.; Dall'Asta, M.; Brighenti, F. Polyphenolic Composition of Hazelnut Skin. J. Agric. Food Chem. 2011, 59, 9935-9941. [CrossRef]

2. Pelvan, E.; Olgun, E.Ö.; Karadağ, A.; Alasalvar, C. Phenolic profiles and antioxidant activity of Turkish Tombul hazelnut samples (natural, roasted, and roasted hazelnut skin). Food Chem. 2018, 244, 102-108. [CrossRef] [PubMed]

3. Piccinelli, A.L.; Pagano, I.; Esposito, T.; Mencherini, T.; Porta, A.; Petrone, A.M.; Gazzerro, P.; Picerno, P.; Sansone, F.; Rastrelli, L.; et al. HRMS Profile of a Hazelnut Skin Proanthocyanidin-rich Fraction with Antioxidant and Anti-Candida albicans Activities. J. Agric. Food Chem. 2016, 64, 585-595. [CrossRef]

4. FAOSTAT. Available online: http:/ / www.fao.org/faostat/en/\#data/QC (accessed on 26 March 2021). 
5. $\quad$ Özdemir, K.S.; Yilmaz, C.; Durmaz, G.; Gökmen, V. Hazelnut skin powder: A new brown colored functional ingredient. Food Res. Int. 2014, 65, 291-297. [CrossRef]

6. Shahidi, F.; Alasalvar, C.; Liyana-Pathirana, C.M. Antioxidant Phytochemicals in Hazelnut Kernel (Corylus avellane L.) and Hazelnut Byproducts. J. Agric. Food Chem. 2007, 55, 1212-1220. [CrossRef]

7. Monagas, M.; Garrido, I.; Lebrón-Aguilar, R.; Bartolome, B.; Gómez-Cordovés, C. Almond (Prunus dulcis (Mill.) D.A. Webb) Skins as a Potential Source of Bioactive Polyphenols. J. Agric. Food Chem. 2007, 55, 8498-8507. [CrossRef]

8. Savic, I.M.; Nikolic, I.L.; Savic-Gajic, I.M.; Kundakovic, T.D. Modeling and optimization of bioactive compounds from chickpea seeds (Cicer arietinum L.). Sep. Sci. Technol. 2019, 54, 837-846. [CrossRef]

9. Makris, D.P.; Boskou, G.; Andrikopoulos, N.K. Recovery of antioxidant phenolics from white vinification solid by-products employing water/ethanol mixtures. Bioresour. Technol. 2007, 98, 2963-2967. [CrossRef] [PubMed]

10. Abbott, A.P.; Capper, G.; Davies, D.L.; Rasheed, R.K.; Tambyrajah, V. Novel solvent properties of choline chloride/urea mixtures. Chem. Commun. 2003, 70-71. [CrossRef]

11. Wang, M.; Wang, J.; Zhang, Y.; Xia, Q.; Bi, W.; Yang, X.; Chen, D.D.Y. Fast environment-friendly ball mill-assisted deep eutectic solvent-based extraction of natural products. J. Chromatogr. A 2016, 1443, 262-266. [CrossRef]

12. Longo, L.S., Jr.; Marcus, V.; Craveiro, B. Deep Eutectic Solvents as Unconventional Media for Multicomponent Reactions. J. Braz. Chem. Soc. 2018, 29, 1999-2025. [CrossRef]

13. Tang, W.; An, Y.; Row, K.H. Emerging applications of (micro) extraction phase from hydrophilic to hydrophobic deep eutectic solvents: Opportunities and trends. TrAC Trends Anal. Chem. 2021, 136, 116187. [CrossRef]

14. Hayyan, M.; Looi, C.Y.; Hayyan, A.; Wong, W.F.; Hashim, M.A. In Vitro and In Vivo Toxicity Profiling of Ammonium-Based Deep Eutectic Solvents. PLoS ONE 2015, 10, e0117934. [CrossRef] [PubMed]

15. Halder, A.K.; Cordeiro, M.N.D.S. Probing the Environmental Toxicity of Deep Eutectic Solvents and Their Components: An In Silico Modeling Approach. ACS Sustain. Chem. Eng. 2019, 7, 10649-10660. [CrossRef]

16. Ruesgas-Ramón, M.; Figueroa-Espinoza, M.C.; Durand, E. Application of Deep Eutectic Solvents (DES) for Phenolic Compounds Extraction: Overview, Challenges, and Opportunities. J. Agric. Food Chem. 2017, 65, 3591-3601. [CrossRef]

17. Meng, Z.; Zhao, J.; Duan, H.; Guan, Y.; Zhao, L. Green and efficient extraction of four bioactive flavonoids from Pollen Typhae by ultrasound-assisted deep eutectic solvents extraction. J. Pharm. Biomed. Anal. 2018, 161, 246-253. [CrossRef]

18. Lakka, A.; Grigorakis, S.; Karageorgou, I.; Batra, G.; Kaltsa, O.; Bozinou, E.; Lalas, S.; Makris, D.P. Saffron Processing Wastes as a Bioresource of High-Value Added Compounds: Development of a Green Extraction Process for Polyphenol Recovery Using a Natural Deep Eutectic Solvent. Antioxidants 2019, 8, 586. [CrossRef]

19. Fanali, C.; Della Posta, S.; Dugo, L.; Russo, M.; Gentili, A.; Mondello, L.; De Gara, L. Application of deep eutectic solvents for the extraction of phenolic compounds from extra-virgin olive oil. Electrophoresis 2020, 41, 1752-1759. [CrossRef]

20. Khezeli, T.; Daneshfar, A.; Sahraei, R. A green ultrasonic-assisted liquid-liquid microextraction based on deep eutectic solvent for the HPLC-UV determination of ferulic, caffeic and cinnamic acid from olive, almond, sesame and cinnamon oil. Talanta 2016, 150, 577-585. [CrossRef]

21. Ozturk, B.; Parkinson, C.; Gonzalez-Miquel, M. Extraction of polyphenolic antioxidants from orange peel waste using deep eutectic solvents. Sep. Purif. Technol. 2018, 206, 1-13. [CrossRef]

22. Fanali, C.; Della Posta, S.; Dugo, L.; Gentili, A.; Mondello, L.; De Gara, L. Choline-chloride and betaine-based deep eutectic solvents for green extraction of nutraceutical compounds from spent coffee ground. J. Pharm. Biomed. Anal. 2020, $189,113421$. [CrossRef]

23. Bonacci, S.; Di Gioia, M.L.; Costanzo, P.; Maiuolo, L.; Tallarico, S.; Nardi, M. Natural Deep Eutectic Solvent as Extraction Media for the Main Phenolic Compounds from Olive Oil Processing Wastes. Antioxidants 2020, 9, 513. [CrossRef]

24. Hayyan, A.; Mjalli, F.S.; AlNashef, I.M.; Al-Wahaibi, T.; Al-Wahaibi, Y.M.; Hashim, M.A. Fruit sugar-based deep eutectic solvents and their physical properties. Thermochim. Acta 2012, 541, 70-75. [CrossRef]

25. Kareem, M.A.; Mjalli, F.S.; Hashim, M.A.; Al Nashef, I.M. Phosphonium-Based Ionic Liquids Analogues and Their Physical Properties. J. Chem. Eng. Data 2010, 55, 4632-4637. [CrossRef]

26. Horinaka, J.-I.; Okamoto, A.; Takigawa, T. Rheological properties of concentrated solutions of gelatin in an ionic liquid 1-ethyl-3methylimidazolium dimethyl phosphate. Int. J. Biol. Macromol. 2016, 91, 789-793. [CrossRef] [PubMed]

27. Nasser, M.S.; Onaizi, S.A.; Hussein, I.A.; Saad, M.A.; Al-Marri, M.J.; Benamor, A. Intercalation of ionic liquids into bentonite: Swelling and rheological behaviors. Colloids Surf. A Physicochem. Eng. Asp. 2016, 507, 141-151. [CrossRef]

28. Al-Sadat, W.; Nasser, M.S.; Chang, F.; Nasr-El-Din, H.; Hussein, I.A. Laboratory evaluation of the effects of additives and pH on the thermorheological behavior of a viscoelastic zwitterionic surfactant used in acid stimulation. J. Pet. Sci. Eng. 2014, 122, 458-467. [CrossRef]

29. Volf, I.; Ignat, I.; Neamtu, M.; Popa, V.I. Thermal stability, antioxidant activity, and photo-oxidation of natural polyphenols. Chem. Pap. 2014, 68, 121-129. [CrossRef]

30. Achkar, T.E.; Fourmentin, S.; Greige-Gerges, H. Deep eutectic solvents: An overview on their interactions with water and biochemical compounds. J. Mol. Liq. 2019, 288, 111028. [CrossRef]

31. Dai, Y.; Witkamp, G.-J.; Verpoorte, R.; Choi, Y.H. Tailoring properties of natural deep eutectic solvents with water to facilitate their applications. Food Chem. 2015, 187, 14-19. [CrossRef] 
32. Lanjekar, K.J.; Rathod, V.K. Green extraction of Glycyrrhizic acid from Glycyrrhiza glabra using choline chloride based natural deep eutectic solvents (NADESs). Process Biochem. 2021, 102, 22-32. [CrossRef]

33. Alcalde, R.; Gutiérrez, A.; Atilhan, M.; Aparicio, S. An experimental and theoretical investigation of the physicochemical properties on choline chloride-Lactic acid based natural deep eutectic solvent (NADES). J. Mol. Liq. 2019, 290, 110916. [CrossRef]

34. Cui, Q.; Peng, X.; Yao, X.-H.; Wei, Z.-F.; Luo, M.; Wang, W.; Zhao, C.-J.; Fu, Y.-J.; Zu, Y.-G. Deep eutectic solvent-based microwave-assisted extraction of genistin, genistein and apigenin from pigeon pea roots. Sep. Purif. Technol. 2015, 150, 63-72. [CrossRef]

35. Jakopic, J.; Petkovsek, M.M.; Likozar, A.; Solar, A.; Stampar, F.; Veberic, R. HPLC-MS identification of phenols in hazelnut (Corylus avellana L.) kernels. Food Chem. 2011, 124, 1100-1106. [CrossRef]

36. Slatnar, A.; Petkovsek, M.M.; Stampar, F.; Veberic, R.; Solar, A. HPLC-MSn identification and quantification of phenolic compounds in hazelnut kernels, oil and bagasse pellets. Food Res. Int. 2014, 64, 783-789. [CrossRef]

37. Yuan, B.; Lu, M.; Eskridge, K.M.; Isom, L.D.; Hanna, M.A. Extraction, identification, and quantification of antioxidant phenolics from hazelnut (Corylus avellana L.) shells. Food Chem. 2018, 244, 7-15. [CrossRef] [PubMed]

38. Skulcova, A.; Russ, A.; Jablonsky, M.; Sima, J. The pH Behavior of Seventeen Deep Eutectic Solvents. BioResources 2018, 13, 5042-5051. [CrossRef]

39. Everette, J.D.; Bryant, Q.M.; Green, A.M.; Abbey, Y.A.; Wangila, G.W.; Walker, R.B. Thorough Study of Reactivity of Various Compound Classes toward the Folin-Ciocalteu Reagent. J. Agric. Food Chem. 2010, 58, 8139-8144. [CrossRef] 